\title{
Distorted Cognitive Processing in Youth: The Structure of Negative Cognitive Errors and Their Associations with Anxiety
}

\author{
Marija Maric • David A. Heyne • \\ Brigit M. van Widenfelt $\cdot$ P. Michiel Westenberg
}

Published online: 19 January 2010

(C) The Author(s) 2010. This article is published with open access at Springerlink.com

\begin{abstract}
The Children's Negative Cognitive Error Questionnaire (CNCEQ) is commonly used to measure four errors in young people's thinking, but research has failed to support the factorial validity of the measure. The primary objective of the present study was to examine the factor structure of a refined and extended version of the CNCEQ. Revision of the CNCEQ involved the exclusion of items rated as contaminated, and the addition of items measuring cognitive errors closely associated with anxiety ('threat conclusion' and 'underestimation of the ability to cope'). A secondary objective was to determine the relation between the negative cognitive errors and anxiety. Principal component analysis of data from 481 children and adolescents indicated five distinct negative cognitive error subscales labeled 'underestimation of the ability to cope', 'personalizing without mind reading', 'selective abstraction', 'overgeneralizing', and 'mind reading' which contained the new 'threat conclusion' items. Confirmatory factor analysis in an independent sample of 295 children and adolescents yielded further support for the five-factor solution. All cognitive errors except 'selective abstraction' were correlated with anxiety. Multiple regression analysis indicated that the strongest predictors of anxiety were the two subscales containing new items, namely 'underestimation of the ability to cope' and 'mind reading'. The results are discussed with respect to further development of
\end{abstract}

M. Maric $(\bowtie)$ D. A. Heyne · P. M. Westenberg Institute of Psychology, Developmental Psychology Unit, Leiden University, P.O. Box 9555, 2300 RB Leiden, The Netherlands e-mail: mmaric@fsw.leidenuniv.nl

B. M. van Widenfelt

Department of Child and Adolescent Psychiatry, Curium-

LUMC/Leiden University Medical Center, Leiden,

The Netherlands the instrument so as to advance the assessment of distorted cognitive processing in young people with internalizing symptoms.

Keywords Cognitive processing .

Negative cognitive errors - Anxiety · Children .

Adolescents

Distorted cognitive processing is important to theoretical and clinical formulations of anxiety and depression in young people and it is often targeted during cognitivebehavioural interventions (e.g., Barrett et al. 2000; Brent and Poling 1997; Curry et al. 2005; Stallard 2005). Assessment instruments have been developed to measure distorted cognitive processing in depressed youth (e.g., the Children's Attributional Style Questionnaire Revised, Thompson et al. 1998; the Children's Dysfunctional Attitude Scale, Abela and Sullivan 2003) and in anxious youth (e.g., Barrett et al. 1996; Bögels and Zigterman 2000). Most of these instruments assess only one or two aspects of distorted cognitive processing. For example, the ambiguous situations were used by Barrett et al. (1996) to assess 'threat interpretations' and by Bögels and Zigterman (2000) to assess 'threat interpretations' and 'coping abilities'. An instrument which assesses a broader range of constructs related to distorted cognitive processing is the Children's Negative Cognitive Error Questionnaire (CNCEQ; Leitenberg et al. 1986). The CNCEQ measures distorted processing in the form of four negative cognitive errors: catastrophizing, overgeneralizing, personalizing, and selective abstraction. The errors have been variously identified among depressed young people (e.g., Kempton et al. 1994; Leitenberg, et al. 1986), anxious young people (e.g., Watts and Weems 2006; Weems et al. 2001, 2007), 
and young people experiencing both depression and anxiety (e.g., Barriga et al. 2000; Epkins 2000; Leung and Wong 1998).

The CNCEQ was modelled after the Cognitive Error Questionnaire (CEQ; Lefebvre 1981) for adults, an instrument designed to measure cognitive errors associated with Beck et al. (1979) cognitive theory of depression. For that reason, it might be regarded as surprising that the CNCEQ has been widely used to assess distorted cognitive processing in anxious youth. The CNCEQ does not measure two cognitive errors commonly referred to in cognitive theories of anxiety (e.g., Beck et al. 1985; Mathews and MacLeod 1994; Weems et al. 2007), namely: 'threat conclusion' and 'underestimation of the ability to cope'. Research has indicated that 'threat conclusion' is indeed characteristic of anxious young people (Barrett et al. 1996; Bell-Dolan 1995; Bögels and Zigterman 2000; Creswell et al. 2005; Hadwin et al. 2006; Muris et al. 2000) as is an 'underestimation of the ability to cope' (Bögels and Zigterman 2000; Weems et al. 2007).

The utility of the CNCEQ for advancing theory and practice associated with internalizing problems in young people is also hampered by the limited empirical support for the differentiation of the four errors included in the CNCEQ. Very few studies have examined the psychometric validity of the theoretically-derived cognitive errors supposedly measured by the CNCEQ, even though the instrument is frequently used to measure these cognitive errors. In the initial psychometric study of the CNCEQ (Leitenberg et al. 1986), there was no examination of the instrument's factor structure. Cole and Turner (1993) were the first to investigate the factor structure of the CNCEQ, using a confirmatory factor analytic approach. Contrary to expectations, support was found for a model which included just one general factor. Subsequently Messer et al. (1994) investigated the factorial validity of the CNCEQ using a principal components analysis (PCA). One of four empirically-derived components accounted for the majority of the variance, items loaded on multiple components, and the components did not correspond with hypothesized item groupings. The researchers ultimately opted for a single factor solution. More recently, Stewart et al. (2004) evaluated the CNCEQ in a community sample of adolescents from the USA and Hong Kong. Consistent with the previous studies, factor analysis indicated that all 24 items loaded onto a single factor. The only study proffering empirical support for separate cognitive errors is that of Karakaya et al. (2007), which is based on a Turkish version of the CNCEQ. Using PCA the authors identified three components which they labeled 'catastrophizing', 'personalizing', and 'selective abstraction'. Items associated with the fourth cognitive error in the CNCEQ (i.e., 'overgeneralizing') loaded on the other three components. Several reported loadings did not approach the minimal level recommended for PCAs (i.e., .32, Tabachnick and Fidell 2007), and there was insufficient information provided about the cross-loadings. In short, factor analyses have not supported the presence of the separate negative cognitive errors presumably measured by the CNCEQ. Further, Weems et al. (2007) reported high and significant correlations (i.e., >.60) between the cognitive error subscales, pointing to overlap across these cognitive constructs.

The relative lack of research support for the existence of distinct cognitive errors in the CNCEQ may be explained by several factors. In line with the notion of negative cognitivity (e.g., Messer et al. 1994), it is possible that cognitive errors are not differentially related to the narrowband conceptualizations of internalizing behaviour. Indeed, studies which have examined relationships between cognitive errors and anxiety and depression have yielded mixed findings, which may be regarded as support for the notion of negative cognitivity. For example, Weems et al. (2001) found that 'catastrophizing' was one of the strongest predictors of anxiety but not of depression, whereas Epkins (1996) found that there was no difference between anxious and depressed groups with respect to 'catastrophizing'. Similarly, 'overgeneralizing' has been found to be predictive of anxiety and depression (Weems et al. 2001), of anxiety alone (Epkins 1996), and of depression alone (Leitenberg et al. 1986). On the other hand, some consistent findings have emerged with respect to the presence of specific cognitive errors in anxiety versus depression. 'Selective abstraction' is more commonly associated with depression than with anxiety (e.g., Leitenberg et al. 1986; Weems et al. 2001, 2007), and 'personalizing' seems to be predictive of anxiety and not of depression (e.g., Epkins 1996; Weems et al. 2001).

Another possible explanation for the lack of research support for the existence of distinct cognitive errors relates to the construction of the CNCEQ. The items designed to measure specific errors may lack specificity. That is, the "thought" intended to reflect a specific cognitive error (e.g., "They probably won't pick me either" to measure overgeneralizing) may bear some resemblance to another cognitive error (e.g., personalizing). Indeed, the clinical practice of cognitive therapy supports the idea that the cognitive errors are not always conceptually discrete (e.g., Wills 2009). Not surprisingly then, when those involved in the development of the adult CEQ tried to assign thoughts to the seven cognitive error categories of Beck et al. (1979), considerable overlap between error categories was observed. Consequently some of the errors were combined, resulting in the four negative cognitive error categories purportedly measured by the CEQ and the 
CNCEQ. ${ }^{1}$ While the combination of cognitive error categories enhanced raters' discrimination among the smaller number of error categories, it may also have reduced the specificity of the resulting cognitive error constructs.

Despite its shortcomings, the CNCEQ has shown promise as a sensitive measure of treatment-related change in the cognitive processing of depressed young people (Kolko et al. 2000) and anxious young people (Silverman et al. 1999). Nevertheless, in its current form the CNCEQ appears inadequate to answer calls for improved cognitive models of the development, maintenance, and treatment of psychopathology in youth. In particular, there is still a need to determine the overlapping and distinctive cognitive factors associated with narrow-band conceptualizations of psychopathology such as anxiety versus depression (Abela and Hankin 2008; Epkins 2000; Messer et al. 1994; Weems et al. 2001). Support for the existence of unique cognitive phenomena represented by specific cognitive errors could provide more direction for cognitive therapists addressing the distorted cognitive processing of their clients (Yurica and DiTomasso 2005), including young clients (Alfano et al. 2002; Kempton, et al. 1994). Over a decade ago Messer et al. (1994) argued for the need to refine the CNCEQ, and to date, this task has not been undertaken. Further development of the CNCEQ ought to focus upon the lack of empirical support for the presence of the four current theoretically derived cognitive errors, and the absence of items to assess errors now commonly associated with anxiety in youth.

The primary objective of the current study was to determine whether a refinement and extension of the CNCEQ would lead to the identification of separate factors representing hypothesized cognitive error categories. This would involve the exclusion of items which appear to reflect multiple cognitive errors and the addition of items which measure 'threat conclusion' and 'underestimation of the ability to cope'. It was anticipated that the revisions made to the CNCEQ would have consequences for the factor structure of the instrument, whereby items assessing distinct cognitive error categories would load on separate components. The internal consistency, intra- and inter-item correlations, inter-subscale correlations, and test-retest reliability of the revised CNCEQ were also investigated. The secondary objective was to examine the extent to which the newly added negative cognitive errors associated with anxiety indeed predict anxiety. In line with the cognitive theory of anxiety (e.g., Beck et al. 1985) and

\footnotetext{
${ }^{1}$ Overgeneralizing' resulted from the combination of Beck et al.'s (1979) 'overgeneralizing' and 'presuming temporal causality'; 'personalizing' was a combination of 'excessive responsibility' and 'selfreference'; 'selective abstraction' was a combination of 'selective abstraction' and 'dichotomous thinking'; and 'catastrophizing' was able to be maintained as an independent category (i.e., without merging it with other categories).
}

empirical research (e.g., Bögels and Zigterman 2000) we expected that the cognitive errors 'threat conclusion' and 'underestimation of the ability to cope' would account for a significant amount of the variance in anxiety.

\section{Methods}

Participants and Procedure

A revised version of the CNCEQ (CNCEQ-R; described below) was administered to two samples of young people (sample $1, N=514$; sample $2, N=304$ ) drawn from nine elementary and secondary public schools in the southwest of the Netherlands. The children and adolescents in sample 1 also completed a measure of anxiety. Participants with any amount of missing data on study measures were removed from the sample (Tabachnick and Fidell 2007), yielding final samples of 481 (sample 1) and 295 (sample 2). In sample 1, participants were aged between 9 and 18 years $(M=14.27 ; \mathrm{SD}=1.79)$ and $58 \%$ were girls. The majority $(69 \%)$ had a Dutch background, $6.4 \%$ were $\mathrm{Su}-$ rinamese, $6.2 \%$ were Antillean, $3.7 \%$ were Turkish, $2.5 \%$ were Moroccan and $11.4 \%$ were of other ethnic backgrounds. Sample 2 included participants aged between 9 and 17 years $(M=13.14$; $\mathrm{SD}=2.10)$ and $52 \%$ were boys. The majority $(89 \%)$ had a Dutch background, $2 \%$ were Surinamese, $1.4 \%$ were Turkish, $1.4 \%$ were Moroccan and $5.4 \%$ were of other ethnic backgrounds.

The study was carried out according to the regulations and with the approval of the Psychology Ethics Committee of the University. Participation was voluntary and anonymous. Parental consent was required as was participants' assent. The five children and adolescents without parental consent were not administered the measures. Participants were administered a booklet of measures in a classroom setting during a free period in school or after school hours. A teacher and at least one masters-level psychology student were present in the classroom at the time of assessment. In sample 1, a measure of anxiety was administered after administration of the CNCEQ-R. Three weeks after the first administration 35 participants from sample 1 completed the CNCEQ-R for a second time, enabling measurement of the test-retest reliability of the measure.

\section{Measures}

\section{Children's Negative Cognitive Error Questionnaire- Revised}

A revised version of the CNCEQ was derived as follows. Firstly, permission to prepare a Dutch, adapted version of the 24-item CNCEQ was obtained from the original author 
(Leitenberg, personal communication, Dec 15, 2005). Procedures for the translation and cross-cultural adaptation of the measure followed those recommended by van Widenfelt et al. (2005). ${ }^{2}$ Secondly, eight new items were developed, four of which were intended to measure 'threat conclusion' (i.e., interpretation of ambiguous situations as threatening) and four intended to measure 'underestimation of the ability to cope' (i.e., the tendency to judge oneself as unable to cope with potentially threatening situations). These items were developed by a team comprising masterand bachelor-level psychology students under the supervision of the first author. The format for each new item followed the format used for the items in the original CNCEQ. A 2- to 3-line description of a hypothetical situation was followed by a statement in the form of a thought about the situation. The thought reflected the cognitive error intended to be measured by the specific item. Participants were asked to imagine that the situation is happening to them and then to rate how similar the associated thought is to how they would think in that situation, using a 5-point scale from "not at all like I would think" (1) to "almost exactly like I would think" (5). The hypothetical situations developed for the 'threat conclusion' items revolved around social threat (three items) or physical threat (one item). For example: You are giving a talk in your class at school. You have just begun when some of your classmates suddenly start to laugh. The associated thought to be rated by subjects is: "They think I am not doing a good job". The situations for the "underestimation of the ability to cope' items covered various content areas: swimming with others, being busy with academic and social activities, moving home and changing school, and social interaction while shopping. For example: Because you are moving, you will go to a different school after the summer, make new friends and get used to a new place. The associated thought is: "I will not be able to handle all these new things." Following Leitenberg et al. (1986), situations associated with family life were avoided to prevent a sense of intrusiveness for subjects (or their parents). Newly developed items were interspersed among the items of the original CNCEQ in a random order. Thirdly, the interim 32-item questionnaire was piloted in a sample of 15 young people between 10 and 18 years of age, testing for the readability of the items. The level of imaginability (i.e., whether children could imagine being in a certain situation) was also assessed. The qualitative responses of piloting subjects lead to several minor changes in the wording of the items.

\footnotetext{
2 We thank our colleagues from University of Amsterdam, Professor Pier J.M. Prins, B.M. Meulema and L.N. Houwer, for their initial work on the translation of the CNCEQ items.
}

Finally, the first and second authors inspected each of the 32 items in order to identify items warranting exclusion on the basis of three decision rules. First, an item was excluded if it was not clearly representative of the intended cognitive error. An example of an item excluded on the basis of the first decision rule is item 10 from the original CNCEQ: You went to a party with one of your friends. When you first got there your friend hung around with some other kids instead of you. Later you and your friend decide to stop at his/her house for a snack before you go home. Later that night you think, "My friend didn't seem to want to hang around with me tonight." This item, intended to measure 'selective abstraction', was excluded because positive experiences with the friend were insufficiently represented in the hypothetical situation to make it a clear case of selective abstraction. Second, an item was excluded if it simultaneously represented more than one of the six cognitive errors (i.e., 'catastrophizing', 'overgeneralizing', 'personalizing', 'selective abstraction', 'threat conclusions' and 'underestimation of the ability to cope'). An example of an item excluded on the basis of the second decision rule is item 22 from the original CNCEQ: Your cousin calls you to ask if you'd like to go on a long bike ride. You think, "I probably won't be able to keep up and people will make fun of me." This item, intended to measure "catastrophizing', was excluded because it also reflected 'underestimation of the ability to cope'. A third decision-rule applied to overlap with cognitive errors beyond the six errors specified above. Almost by definition, 'selective abstraction' incorporates the error of 'black-and-white thinking': "Selective abstraction-the process of exclusively focusing on one negative aspect or detail of a situation, magnifying the importance of that detail, thereby casting the whole situation in a negative context [emphasis added]" (Yurica and DiTomasso 2005, p. 119). Thus, while "selective abstraction' items could reflect 'black-and-white thinking', any other items which contained 'black-and-white thinking' were excluded (e.g., a deleted 'overgeneralizing' item was: Last week you played softball and struck out twice. Today some kids from your class ask you to play soccer. You think, “There's no sense playing, I'm no good at sports."). 'Mind reading' is a cognitive error which has been defined as follows: "One's arbitrary conclusion that someone is reacting negatively, or thinking negatively toward him/her, without specific evidence to support that conclusion" (p. 119). The new items measuring 'threat conclusion' could contain 'mind reading', but items measuring any other error were excluded if they contained 'mind reading' (e.g., a deleted 'catastrophizing' item was: Your softball team is having practice. The coach tells you he would like to talk to you after practice. You think, "He's not happy with how I'm doing and doesn't want me on the team anymore"). Another cognitive error is 'fortune-telling', defined by Yurica and DiTomasso 
(2005) as: "The process of foretelling or predicting the negative outcome of a future event or events and believing this prediction is absolutely true for oneself" (p. 119). Only those CNCEQ items measuring 'overgeneralizing' were permitted to contain 'fortune-telling'. During the rating no items other than the 'overgeneralizing' items were found to contain 'fortune-telling'. Inter-rater agreement on the exclusion and retention of items was $93 \%$ (i.e., agreement on 29 out of 32 items). Disagreements were resolved through consensus discussion.

The final self-report questionnaire comprised 18 items representing the following five theoretically-derived subscales: 'overgeneralizing' (4 items), 'personalizing' (5 items), 'selective abstraction' (3 items), 'threat conclusion' ( 2 items), and 'underestimation of the ability to cope' (4 items). Based on the aforementioned decision rules, all six of the items representing 'catastrophizing' were excluded from the questionnaire; five of these six items contained 'mind reading' and one of the items contained 'underestimation of the ability to cope' ${ }^{3}$

\section{The Screen for Child Anxiety Related Emotional Disorders- Short (SCARED-5)}

The SCARED-5 is a self-report questionnaire based on the 41-item version of the SCARED (Birmaher et al. 1999). The SCARED includes 5 factors that parallel the DSM-IV classification of anxiety disorders: 'panic/somatic', 'generalized anxiety', 'separation anxiety', 'social phobia', and 'simple phobia'. Each item is scored using a 3-point scale (not true or hardly ever true, sometimes true, often true). Because the current study was part of a larger study, the short version of the SCARED (i.e., SCARED-5) was used. The original authors of the SCARED derived the SCARED- 5 by selecting the highest-loading item on each of the five factors. The SCARED-5 has demonstrated good internal consistency and discriminant validity (Birmaher et al. 1999).

\section{Results}

What is the Factor Structure of the CNCEQ-R?

The range and skewness of item scores derived from sample 1 were acceptable for the planned analyses, and there were no outliers in the data. During initial exploratory analysis of the data an additional two items were removed (an original 'overgeneralizing' item and a new

\footnotetext{
${ }^{3}$ English and Dutch versions of the CNCEQ-R are available on request from the first author.
}

'underestimation of the ability to cope' item). These items loaded on components not representative of the intended cognitive error category, and it became evident that the item content overlapped somewhat with other cognitive errors. Principal factors extraction with oblique rotation was then performed with the remaining 16 items of the CNCEQ-R. An oblique rotation was carried out assuming that the components would be correlated (Tabachnick and Fidell 2007). The scree plot resulted in a point of inflexion after the first component suggesting a one component solution. The analysis yielded four components with eigenvalues $>1$ (i.e., $4.02,1.27,1.18,1.07$ ) and a fifth component with an eigenvalue close to 1.00 (i.e., 0.997). These five components accounted for $53.37 \%$ of the total variance. The item loadings are presented in Table 1. Items intended to measure 'underestimation of the ability to cope', 'selective abstraction', and 'overgeneralizing' loaded on distinct components. Three of the five 'personalizing' items loaded onto a distinct component, while the other two 'personalizing' items loaded on a component together with the two 'threat conclusion' items. The four items in this latter component all reflected the cognitive error of 'mind reading', whereas the three 'personalizing' items loading on a separate component did not reflect the cognitive error of 'mind reading'. The five components were thus labeled: 1 -underestimation of the ability to cope (UAC), 2-personalizing without mind reading (PER), 3-selective abstraction (SA), 4-overgeneralizing $(\mathrm{OV})$, and 5-mind reading (MR). All items loaded on the respective components at .40 or above, with no crossloadings above 40 .

Given that the eigenvalue for one of the components was slightly less than 1 , a four-factor solution was also investigated. The 'underestimation of the ability to cope' items and Leitenberg et al. (1986) original 'personalizing' items merged within one and the same component. Furthermore, the 'personalizing' items had component loadings around .30 and loaded on more than two components. Thus, the four factor solution was considered to be less sound, theoretically and statistically, relative to the five factor solution.

In an attempt to replicate the solution, a five-factor model was tested via confirmatory factor analysis (CFA) of the 16 items of the CNCEQ-R. The data derived from sample 2 was used for the CFA. No outliers were found, and item scores had acceptable range and skewness. CFA was performed using EQS 6.1 for Windows (Bentler 2002). The model was computed using Maximum Likelihood estimation. A number of fit indices and conventions were examined to test the five-factor model: The comparative fit index (CFI), the goodness of fit index (GFI), and the root mean-square error of approximation (RMSEA) (Hu and Bentler 1999). CFI and GFI values above .90 
Table 1 Component loadings of the CNCEQ-R items (PCA, oblique rotation)

\begin{tabular}{|c|c|c|c|c|c|}
\hline \multirow[t]{2}{*}{ Abbreviated description of item-related hypothetical situation (type of error; content area) } & \multicolumn{5}{|c|}{ Component } \\
\hline & 1 & 2 & 3 & 4 & 5 \\
\hline 14. Coping with being very busy with school and social tasks (UAC ${ }^{b}$; academic/social) & .832 & .011 & -.037 & -.099 & -.16 \\
\hline 16. Coping with a move involving a new school and friends $\left(\mathrm{UAC}^{\mathrm{b}}\right.$; general) & 619 & -.007 & .126 & -.109 & .03 \\
\hline 7. Coping with feeling awful after somebody is unkind to you (UAC ${ }^{\mathrm{b}}$; general/social) & .552 & -.065 & -.168 & .030 & .31 \\
\hline 1. Your team loses a 4-person relay race $\left(\mathrm{PER}^{\mathrm{a}}\right.$; athletic) & .105 & -.759 & .041 & -.025 & -.059 \\
\hline 3. Your team loses a spelling contest (PER ${ }^{\mathrm{a}}$; academic) & -.004 & -.628 & -.038 & -.046 & .29 \\
\hline 6. You get a poor grade for a group science project $\left(\mathrm{PER}^{\mathrm{a}}\right.$; academic) & -.094 & -.551 & .150 & -.352 & -.01 \\
\hline 9. You play basketball and score five shots and miss two easy shots $\left(\mathrm{SA}^{\mathrm{a}}\right.$; athletic) & .022 & -.173 & .734 & .183 & .075 \\
\hline $\begin{array}{l}\text { 8. You were having a good day at school until the last hour when you did poorly on a quiz } \\
\left(\mathrm{SA}^{\mathrm{a}} \text {; academic) }\right.\end{array}$ & -.148 & .249 & .590 & -.311 & -.013 \\
\hline 2. You try out for softball team and get two hits and make two outs $\left(\mathrm{SA}^{\mathrm{a}}\right.$; athletic) & .218 & -.315 & .497 & .085 & -.087 \\
\hline $\begin{array}{l}\text { 4. Some friends ask if you will try out for the school volleyball team; you tried last year and didn't } \\
\text { make it }\left(\mathrm{OV}^{\mathrm{a}} \text {; athletic }\right)\end{array}$ & .019 & -.215 & -.148 & -.654 & -.012 \\
\hline 13. Your class is starting a new unit in math and the last one was very hard $\left(\mathrm{OV}^{\mathrm{a}}\right.$; academic) & .232 & .085 & -.005 & -.625 & -.013 \\
\hline $\begin{array}{l}\text { 11. You forgot some things during a history test last week and now you are having a math test } \\
\left(\mathrm{OV}^{\mathrm{a}} \text {; academic) }\right.\end{array}$ & .013 & -.068 & .114 & -.615 & .172 \\
\hline $\begin{array}{l}\text { 5. You call a friend to talk about homework and the friend says he/she can't talk to you now } \\
\text { because someone needs the phone (PER }{ }^{\mathrm{a}} \text {; social) }\end{array}$ & -.132 & -.031 & -.103 & -.019 & .85 \\
\hline $\begin{array}{l}\text { 10. You bought new shoes and when you wear them your friends ask if you have new shoes } \\
\text { (TC }{ }^{\text {b }} \text { social) }\end{array}$ & .223 & .246 & .230 & -.202 & .46 \\
\hline 15. You are giving a talk to your class and some of your classmates start laughing ( $\mathrm{TC}^{\mathrm{b}}$; social) & .333 & -.098 & .204 & .069 & .45 \\
\hline $\begin{array}{l}\text { 12. You are taking skiing lessons and the instructor tells the group that some people are not ready } \\
\text { for the steep trails yet (PER }{ }^{\mathrm{a}} \text {; athletic) }\end{array}$ & .098 & -.287 & .103 & -.099 & \\
\hline
\end{tabular}

Loadings $>.40$ are shown in bold. Component $1=$ underestimation of the ability to cope (UAC); Component $2=$ personalizing without mind reading (PER); Component $3=$ selective abstraction (SA); Component $4=$ overgeneralizing (OV); Component $5=$ mind reading (MR)

a Original CNCEQ items: 'personalizing' (PER), 'selective abstraction' (SA), and 'overgeneralizing' (OV)

b Newly added items: 'underestimation of the ability to cope' (UAC) and 'threat conclusion' (TC)

suggest a good model fit (Kline 2005), and an RMSEA value below .05 indicates an excellent model fit (Browne and Cudeck 1993).

The results indicated that the five-factor model represented an overall good fit to the data, with GFI and CFI indices indicating a good fit, and RMSEA indicating an excellent fit: $\mathrm{CFI}=.93, \mathrm{GFI}=.95$, and RMSEA $=.04 \mathrm{~A}$ one-factor solution was also examined, given that previous studies of the factor structure of the CNCEQ suggested the presence of a general dimension of cognitive processing as opposed to distinguishable cognitive errors. The fit of the one-factor model was less adequate than the fit of the fivefactor model $(\mathrm{CFI}=.84, \mathrm{GFI}=.91$, and RMSEA $=.06)$. The results indicated that, although one of the three indices for the one-factor model was acceptable (i.e., GFI), the indices for the five-factor model were superior. The results of the CFA provided support for the factor structure of the CNCEQ-R scores established through the PCA.

In sample 1, internal consistency (Cronbach's alpha) for the total CNCEQ-R score was .80. The alphas for the five subscales were $.58, .60, .35, .53$, and .62 , respectively. It should be noted that the number of items in the subscales is small (i.e., 3 or 4), possibly accounting for the lower alphas for the subscales. Correlation analyses were used to determine intra-item relatedness (i.e., among the items that make up each subscale) and inter-item relatedness (i.e., between the items of different subscales) (Clark and Watson 1995). Mean intra-item correlations for each subscale fell inside the range $0 \pm .10-0 \pm .50$, representing an acceptable level of homogeneity (Nunnally 1978). The mean intra-item correlations were: 32 for 'underestimation of the ability to cope' items, .33 for 'personalizing without mind reading' items, .15 for 'selective abstraction' items, .27 for 'overgeneralizing' items, and .29 for 'mind reading' items. All mean intra-item correlations were higher than the mean inter-item correlations for the five subscales (.18, $.19, .13, .17$, and .17 , respectively). Means and standard deviations for the five subscales are presented in Table 2, together with the correlations between the five subscales. The correlations between the subscales were all positive and ranged from medium to small.

Test-retest reliability analyses indicated that the five subscales were moderately stable over time, with Pearson correlation coefficients of .77, .85, .71, .82, and .74, 
Table 2 Descriptives and Pearson correlations for the five negative cognitive error subscales

\begin{tabular}{lllllll}
\hline & 1 & 2 & 3 & 4 & 5 & $\begin{array}{l}\text { Mean } \\
(\mathrm{SD})\end{array}$ \\
\hline (1) UAC & - & & & & & $1.99(.78)$ \\
(2) PER & .31 & - & & & & $2.12(.84)$ \\
(3) SA & .24 & .31 & - & & & $2.06(.76)$ \\
(4) OV & .36 & .34 & .25 & - & & $2.02(.86)$ \\
(5) MR & .48 & .41 & .31 & .41 & - & $2.08(.77)$ \\
\hline
\end{tabular}

$U A C$ underestimation of the ability to cope, $P E R$ personalizing without mind reading, $S A$ selective abstraction, $O V$ overgeneralizing, $M R$ mind reading

respectively. The correlation coefficient for the test-retest reliability of the total CNCEQ-R score was .90 .

\section{Which Cognitive Errors Predict Anxiety?}

Multiple linear regression analysis was conducted to determine which of the negative cognitive error subscales were the strongest predictors of anxiety. The total SCARED-5 anxiety score was used as the dependant variable and negative cognitive error scores were entered as predictors. $\mathrm{R}$ for regression was significantly different from zero, $F(5,475)=23.99, p<.001$, with an Adjusted $\mathrm{R}^{2}$ at .19. The $\mathrm{R}^{2}$ value of .20 indicates that $20 \%$ of the variability in anxiety was predicted by negative cognitive errors. As can be seen from Table 3, the size and direction of the relationships suggests that higher levels of negative cognitive errors 'mind reading', 'underestimation of the ability to cope', 'overgeneralizing' and 'personalizing' are found among children and adolescents with higher levels of anxiety. Among these four errors, 'mind reading', 'underestimation of the ability to cope', and 'overgeneralizing' were the most important, as indicated by the semipartial correlations. These three error categories had a significant unique contribution in the explanation of anxiety, with 'underestimation of the ability to cope' and 'mind reading' being the two strongest predictors. The cognitive error

Table 3 Summary of multiple regression analysis predicting anxiety

\begin{tabular}{lcrlc}
\hline Variables & \multicolumn{1}{l}{$B$} & \multicolumn{1}{l}{$\beta$} & $r$ & Semipartial r \\
\hline UAC & $.37^{*}$ & .20 & $.34^{*}$ & .17 \\
PER & .00 & .00 & $.18^{*}$ & .00 \\
SA & $-.32^{*}$ & -.15 & .01 & -.14 \\
OV & $.30^{*}$ & .15 & $.29^{*}$ & .13 \\
MR & $.52^{*}$ & .25 & $.37^{*}$ & .20 \\
\hline
\end{tabular}

UAC underestimation of the ability to cope, PER personalizing without mind reading, $S A$ selective abstraction, $O V$ overgeneralizing, $M R$ mind reading

$* p<.01$ 'personalizing without mind reading' seems not to be a unique predictor of anxiety when controlling for all other negative cognitive errors. The direction of the semi-partial correlation for the cognitive error 'selective abstraction' was negative, indicating that higher levels of this error were related to lower levels of anxiety when controlling for the influence of all other errors.

\section{Discussion}

Via the CNCEQ-R-a refinement and extension of Leitenberg et al. (1986) CNCEQ - this study has provided empirical support for separate negative cognitive error categories measuring 'underestimation of the ability to cope', 'personalizing without mind reading', 'selective abstraction', 'overgeneralizing', and 'mind reading'. Previous studies of the factor structure of the 24-item CNCEQ, variously using exploratory (EFA) or confirmatory factor analyses (CFA), have generally converged on the notion that the measure consists of just one component representing negative cognitive processing. The conclusions from these previous studies were based upon the researchers' inspection of the scree plot, the eigenvalues, component loadings, and fit indices. Similar to these previous studies, the scree plot in the current study suggested a one-factor solution. At the same time, the eigenvalues and component loadings associated with the EFA in the current study suggested the presence of multiple components. CFA provided further support for a five-factor solution, and the support for a one-factor solution was less strong. The only other study which has been suggestive of the presence of multiple CNCEQ components was that of Karakaya et al. (2007), which made use of EFA. Whereas the components in Karakaya et al.'s study were somewhat questionable (e.g., they were based on low item loadings and they were not clear representations of the separate cognitive error categories), the current study identified components representative of distinct cognitive error categories and most loadings were far above the Tabachnick and Fidell (2007) criterion. It is likely that the unique results of the current study flow from the close attention paid to the refinement and extension of the original CNCEQ.

In previous studies on the CNCEQ, scores for the cognitive error categories were highly correlated (e.g., Weems et al. 2007). The fact that the correlations among the cognitive error categories of the CNCEQ-R were lower in comparison with previous studies supports the notion that the refinement of the item set has lead to improved differentiation among cognitive errors. At the same time, the correlations among the error categories of the CNCEQ-R were still moderate, suggesting that the error categories are part of a higher order construct of distorted cognitive 
processing. The internal consistency of the CNCEQ-R subscales was low to moderate. This may be due to the small number of items in the cognitive error categories. At the same time, mean intra-item correlations were higher than mean inter-item correlations for the five subscales, suggesting the homogeneity of the specific cognitive error subscales. The test-retest reliability of the CNCEQ-R subscales was moderate.

This study also investigated the relation between the negative cognitive errors in the CNCEQ-R and anxiety. Consistent with previous research, the results indicated that 'selective abstraction' was not associated with anxiety (e.g., Weems et al. 2001, 2007) and that 'overgeneralizing' was associated with anxiety (e.g., Epkins 1996; Weems et al. 2001). Two subscales containing newly added items designed to measure negative cognitive errors presumably characteristic of anxiety (i.e., 'underestimation of the ability to cope' and 'mind reading' which contained the 'threat conclusion' items) were not only significantly associated with anxiety, but were the two strongest predictors of anxiety when controlling for the influence of other error categories. This result is consistent with the notion that certain cognitive errors may be differentially important to the phenomenology of anxiety.

A noteworthy finding pertains to the relationship between anxiety and the cognitive process of personalizing. After the refinement procedure, five of the six original items from Leitenberg et al. (1986) 'personalizing' subscale remained. Three of these items formed the new 'personalizing without mind reading' subscale. For these three items, the thoughts associated with the hypothetical scenarios involve an internal attribution for a negative event (i.e., the negative event would not have occurred "if I had been faster" or "if I were smarter" or "if I didn't do a lousy job"). None of these three items include the additional suggestion of psychological threat (in the form of 'mind reading'), a construct which is often associated with anxiety. Previously, researchers have found medium correlations between the original six-item 'personalizing' subscale and anxiety (Epkins 1996; Weems et al. 2001, 2007), and two studies found that the 'personalizing' subscale significantly added to the prediction of anxiety (Weems et al. 2001, 2007). In the current study, however, the correlation between 'personalizing without mind reading' and anxiety was small, and the 'personalizing without mind reading' subscale did not have a unique contribution to the prediction of anxiety. Notwithstanding the fact that the new subscale has fewer items relative to the original subscale (three versus six), it might be argued that the new subscale is a more pure measure of 'internal attributions for negative events,' a construct which is linked to depression (Abramson et al. 1989). Future efforts to determine the distinct and overlapping cognitive features of anxiety and depression in youth might make use of this type of purer measure of personalizing (i.e., uncontaminated by items which are also associated with psychological threat).

Overall, it appears that the refinement and extension of the original CNCEQ has yielded a measure which has the potential to advance research on cognitive processes associated with internalizing problems in young people. It is timely that newly added items appear to assess separate cognitive constructs associated with anxiety, given that much is still unknown about the role of distorted cognitive processing in children and adolescents with anxiety (Stallard 2009; Weems et al. 2007), and given that conceptual overlap between the cognitive constructs associated with anxiety is regarded as one of the largest limitations of the existing literature (Starcevic and Berle 2006). To further facilitate the study of cognitive processing in anxious youth, new items could be developed to replace the 'catastrophizing' items which were excluded during refinement of the CNCEQ. In addition, to improve the reliability of the CNCEQ-R subscales, extra items should be added to each of the five subscales. Refinement of the CNCEQ also meant that some domains were no longer adequately represented within each subscale (e.g., the 'overgeneralizing' subscale no longer contains an item from the 'social' domain). Thus, the development of new items ought to account for adequate coverage of the domains within each error category. Finally, in line with the current refinement of the CNCEQ, each new item should be a clear representation of the intended negative cognitive error, and it should not overlap with other error categories included in the measure.

Future research should focus on replicating the factor structure found in this study, making use of the 16-item CNCEQ-R as well as an extended version which incorporates more items per subscale. Such replication should also occur in an English-speaking sample of young people. Ultimately, the factor structure of the CNCEQ-R and extended versions should be explored among clinical samples of anxious young people. Moreover, to draw firmer conclusions about the specificity of the negative cognitive error categories for anxious and depressed children and adolescents, the CNCEQ-R should be examined among anxious, depressed, and anxious-depressed young people.

In summary, in response to calls for improved conceptualization and assessment of distorted cognitive processing in anxious young people, we developed a revised version of the CNCEQ. The results from this study suggest that the CNCEQ-R is comprised of five empiricallyderived negative cognitive error categories which are differentially related to anxiety in youth. With improvements in the assessment of cognitive errors in young people can come increased understanding of the role of distorted cognitive processing in anxiety and depression. This is of 
direct relevance for treatment given that the focus of treatment can vary in accordance with cognitive differences between individuals and disorders (Beck 1988). Further, in the same way that the CNCEQ has been found to be a sensitive measure of treatment-related reductions in distorted processing (Kolko et al. 2000; Silverman, et al. 1999), it is likely that the CNCEQ-R will be of substantial importance in measuring cognitive change in clinical and research settings.

Acknowledgments We would like to thank Dr. Caroline L. Bokhorst for her help with conducting the confirmatory factor analysis.

Open Access This article is distributed under the terms of the Creative Commons Attribution Noncommercial License which permits any noncommercial use, distribution, and reproduction in any medium, provided the original author(s) and source are credited.

\section{References}

Abela, J. R. Z., \& Hankin, B. (2008). Cognitive vulnerability to depression in children and adolescents: A developmental psychopathology perspective. In J. R. Z. Abela \& B. L. Hankin (Eds.), Handbook of depression in children and adolescents (pp. 35-78). New York: The Guilford Press.

Abela, J. R. Z., \& Sullivan, C. (2003). A test of Beck's cognitivediathesis theory of depression in early adolescents. Journal of Early Adolescence, 23, 384-404.

Abramson, L. Y., Metalsky, G. L., \& Alloy, L. B. (1989). Hopelessness depression: A theory based subtype of depression. Psychological Review, 96, 358-372.

Alfano, C. A., Beidel, D. C., \& Turner, S. M. (2002). Cognition in childhood anxiety: Conceptual, methodological, and developmental issues. Clinical Psychology Review, 22, 1209-1238.

Barrett, P. M., Rapee, R. M., Dadds, M. M., \& Ryan, S. M. (1996). Family enhancement of cognitive style in anxious and aggressive children. Journal of Abnormal Child Psychology, 24, 187-203.

Barrett, P. M., Lowry-Webster, H., \& Turner, C. (2000). Friends program for youth: Group leaders manual. Brisbane: Australian Academic Press.

Barriga, A. Q., Landau, J. R., Stinson, B. L., I. I., Liau, A. K., \& Gibbs, J. C. (2000). Cognitive distortion and problem behaviours in adolescents. Criminal Justice and Behavior, 27, 36-56.

Beck, A. T. (1988). Cognitive approaches to panic disorder: Theory and therapy. In S. Rachman \& J. D. Maser (Eds.), Panic: Psychological perspectives. Hillsdale, New Jersey: Lawrence Erlbaum.

Beck, A. T., Emery, G., \& Greenberg, R. L. (1985). Anxiety disorders and phobias: A cognitive perspective. New York: Basic Books.

Beck, A. T., Rush, A. J., Shaw, B. F., \& Emery, G. (1979). Cognitive therapy of depression. New York: The Guilford Press.

Bell-Dolan, D. J. (1995). Social cue interpretation of anxious children. Journal of Clinical Child Psychology, 24, 1-10.

Bentler, P. M. (2002). EQS 6 structural equations program manual. Encino, CA: Multivariate Software.

Birmaher, B., Brent, D. A., Chiappetta, L., Bridge, J., Monga, S., \& Baugher, M. (1999). Psychometric properties of the screen for child anxiety related emotional disorders (scared): A replication study. Journal of American Academy of Child and Adolescent Psychiatry, 38, 1230-1236.
Bögels, S. M., \& Zigterman, D. (2000). Dysfunctional cognitions in children with social phobia, separation anxiety disorder, and generalized anxiety disorder. Journal of Abnormal Child Psychology, 28, 205-211.

Brent, D. A., \& Poling, K. (1997). Cognitive therapy treatment manual for depressed and suicidal youth. Pittsburgh, PA: University of Pittsburgh, Services for Teens at Risk.

Browne, M., \& Cudeck, R. (1993). Alternative ways of testing model fit. In K. Bollen \& J. Long (Eds.), Testing structural equation models (pp. 136-162). Newbury Park, CA: Sage.

Clark, L. A., \& Watson, D. (1995). Constructing validity: Basic issues in objective scale development. Psychological Assessment, 7, 309-319.

Cole, D. A., \& Turner, J. E. (1993). Models of cognitive mediation and moderation in child depression. Journal of Abnormal Psychology, 102, 271-281.

Creswell, C., Schniering, C. A., \& Rapee, R. M. (2005). Threat interpretation in anxious children and their mothers: Comparison with nonclinical children and the effects of treatment. Behaviour Research and Therapy, 43, 1375-1381.

Curry, J.F., Wells, K.C., Brent, D.A., Clarke, G.N., Rohde, P., Albano, \& A.M., et al. (2005). Treatment for adolescent depression study (TADS): Cognitive behavior therapy manual. Retrieved March 17, 2009, from Duke University Medical Center, The TADS team Web site: https://trialweb.dcri.duke.edu/ tads/tad/manuals/TADS_CBT.pdf.

Epkins, C. C. (1996). Cognitive specificity and affective confounding in social anxiety and dysphoria in children. Journal of Psychopathology and Behavioral Assessment, 18, 83-101.

Epkins, C. C. (2000). Cognitive specificity in internalizing and externalizing problems in community and clinical-referred children. Journal of Clinical Child Psychology, 29, 199-208.

Hadwin, J. A., Garner, M., \& Perez-Olivas, G. (2006). The development of information processing biases in childhood anxiety: A review and exploration of its origins in parenting. Clinical Psychology Review, 26, 876-894.

Hu, L., \& Bentler, P. M. (1999). Cutoff criteria for fit indices in covariance structure analysis: Conventional criteria versus new alternatives. Structural Equation Modeling, 6, 1-55.

Karakaya, I., Coskun, A., Agaoglu, B., Oc, O. Y., Cakin Memik, N., Sismanlar, S. G., et al. (2007). The reliability and validity of the children's negative cognitive error questionnaire. Turk Psikiyatri Dergisi, 18, 155-162.

Kempton, T., Van Hasselt, V. B., Bukstein, O. G., \& Null, J. A. (1994). Cognitive distortions and psychiatric diagnosis in dually diagnosed adolescents. Journal of the American Academy of Child and Adolescent Psychiatry, 33, 217-222.

Kline, R. B. (2005). Principles and practice of structural equation modeling (2nd ed.). New York: Guilford Press.

Kolko, D. J., Brent, D. A., Baugher, M., Bridge, J., \& Birmaher, B. (2000). Cognitive and family therapies for adolescent depression: Treatment specificity, mediation, and moderation. Journal of Consulting and Clinical Psychology, 68, 603-614.

Lefebvre, M. F. (1981). Cognitive distortion and cognitive errors in depressed psychiatric and low back pain patients. Journal of Consulting and Clinical Psychology, 49, 517-525.

Leitenberg, H., Yost, L. W., \& Carroll-Wilson, M. (1986). Negative cognitive errors in children: Questionnaire development, normative data, and comparisons between children with and without self-reported symptoms of depression, low self-esteem, and evaluation anxiety. Journal of Consulting and Clinical Psychology, 54, 528-536.

Leung, P. W. L., \& Wong, M. M. T. (1998). Can cognitive distortions differentiate between internalising and externalising problems? Journal of Child Psychology and Psychiatry and Allied Disciplines, 39, 263-269. 
Mathews, A., \& MacLeod, C. (1994). Cognitive approaches to emotion and emotional disorders. Annual Review of Psychology, $45,25-50$.

Messer, S. C., Kempton, T., Van Hasselt, V. B., Null, J. A., \& Bukstein, O. G. (1994). Cognitive distortions and adolescent affective disorder. Validity of the CNCEQ in an inpatient sample. Behavior Modification, 118, 339-351.

Muris, P., Lauermans, J., Merckelbach, H., \& Mayer, B. (2000). Danger is lurking everywhere. The relation between anxiety and threat perception abnormalities in normal children. Journal of Behavior Therapy and Experimental Psychiatry, 31, 123-136.

Nunnally, J. (1978). Psychometric theory. McGraw-Hill: New York.

Silverman, W. K., Kurtines, W. M., Ginsburg, G. S., Weems, C. F., Rabian, B., \& Serafini, L. T. (1999). Contingency management, self-control, and education support in the treatment of childhood phobic disorders: A randomized clinical trial. Journal of Consulting and Clinical Psychology, 67, 675-687.

Stallard, P. (2005). A clinician's guide to think good-feel good: Using $C B T$ with children and young people. Chichester: Wiley.

Stallard, P. (2009). Anxiety: Cognitive behavioural therapy with children and young people. London: Bruner Routledge.

Starcevic, V., \& Berle, D. (2006). Cognitive specificity of anxiety disorders: A review of selected key constructs. Depression and Anxiety, 23, 51-61.

Stewart, S., Kennard, B., Lee, P., Hughes, C., Mayes, T., Emslie, G., et al. (2004). A cross-cultural investigation of cognitions and depressive symptoms in adolescents. Journal of Abnormal Psychology, 113, 248-257.

Tabachnick, B. G., \& Fidell, L. S. (2007). Using multivariate statistics (5th ed.). Boston: Allyn and Bacon.
Thompson, M., Kaslow, N. J., Weiss, B., \& Nolen-Hoeksema, S. (1998). Children's attributional style questionnaire revised: Psychometric examination. Psychological Assessment, 10, 166-170.

Van Widenfelt, B. M., Treffers, P. D. A., Beurs, E., Siebelink, B. M., \& Koudijs, E. (2005). Translation and cross-cultural adaptation of assessment instruments used in psychological research with children and families. Clinical Child and Family Psychological Review, 8, 135-147.

Watts, S. E., \& Weems, C. F. (2006). Associations among selective attention, memory bias, cognitive errors and symptoms of anxiety in youth. Journal of Abnormal Child Psychology, 34, 841-852.

Weems, C. F., Berman, S. L., Silverman, W. K., \& Saavedra, L. M. (2001). Cognitive errors in youth with anxiety disorders: The linkages between negative cognitive errors and anxious symptoms. Cognitive Therapy and Research, 25, 559-575.

Weems, C. F., Costa, N. M., Watts, S. E., Taylor, L. K., \& Cannon, M. F. (2007). Cognitive errors, anxiety sensitivity, and anxiety control beliefs-their unique and specific associations with childhood anxiety symptoms. Behavior Modification, 31, 174-201.

Wills, F. (2009). Beck's cognitive therapy: Distinctive features. London: Routledge.

Yurica, C. L., \& DiTomasso, R. A. (2005). Cognitive distortions. In A. Freeman, S. H. Felgoise, C. M. Nezu, A. M. Nezu, \& M. Reinecke (Eds.), International encyclopedia of cognitive and behavioral therapies. New York: Springer. 